UCRL-JC-127767

PREPRINT

\title{
Designs for a High Power Superconducting Delay Line
}

\author{
Y-J. Chen \\ G. Caporaso
}

This paper was prepared for submittal to the 11th IEEE International Pulsed Power Conference

Baltimore, Maryland

June 29-July 2, 1997

June 26, 1997

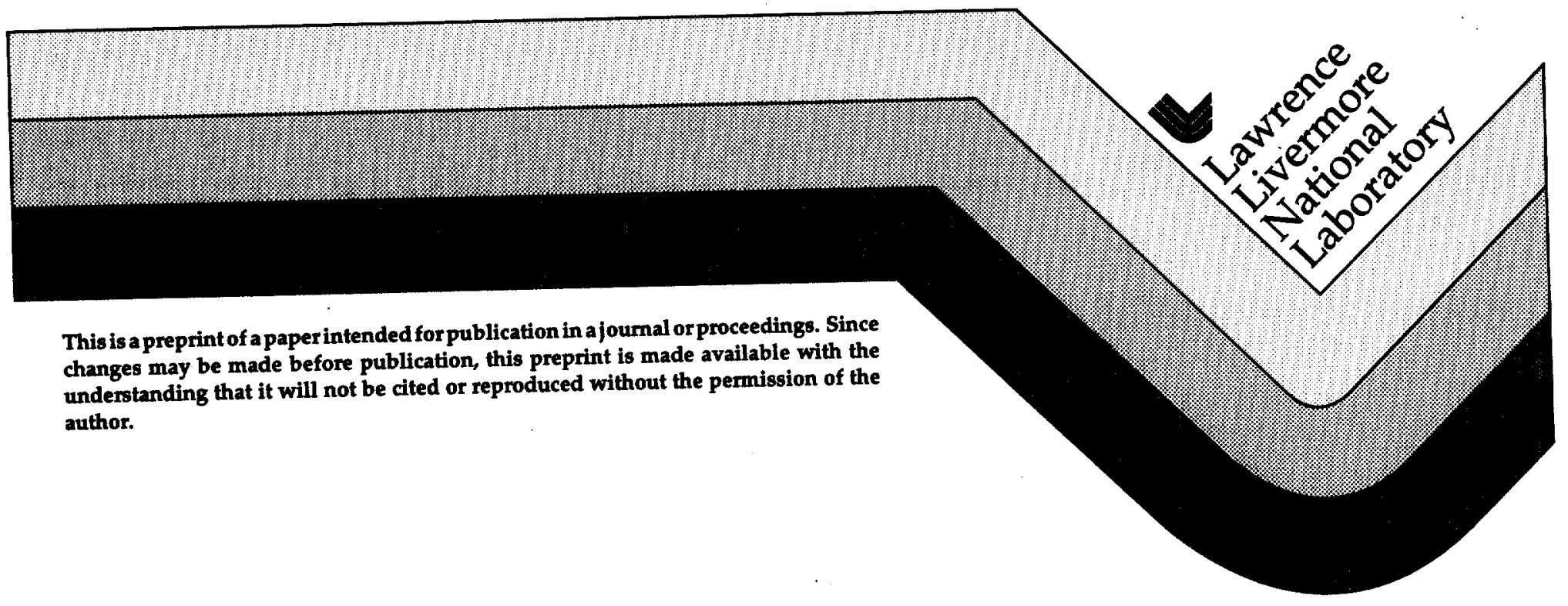




\section{DISCLAIMER}

This document was prepared as an account of work sponsored by an agency of the United States Government. Neither the United States Government nor the University of Califorria nor any of their employees, makes any warranty, express or implied, or assumes any legal liability or responsibility for the accuracy. completeness, or usefulness of any information, apparatus, product, or process disclosed, or represents that its use would not infringe privately owned rights. Reference herein to any specific commercial product, process, or service by trade name, trademark, manufacturer, or otherwise, does not necessarily constitute or imply its endorsement, recommendation, or favoring by the United States Goverrment or the University of California. The views and opinions of authors expressed herein do not necessarily state or reflect those of the United States Government or the University of California, and shall not be used for advertising or product endorsement purposes. 


\title{
DESIGNS FOR A HIGH POWER SUPERCONDUCTING DELAY LINE
}

\author{
Yu Ju (Judy) Chen and G. Caporaso, Lawrence Livermore National Laboratory, University of \\ California, P.O. Box 808, Livermore, CA 94550
}

Abstract

Potential designs for a high power superconducting delay line of approximately $10 \mu \mathrm{s}$ duration are described. The transmitted signal should have low dispersion and little attenuation to recapture the original signal. Such demands cannot be met using conventional metal conductors. This paper outlines a proposal for a new transmission line design using low temperature superconducting material which meets system specifications. The $25 \Omega$ line is designed to carry pulsed signals with an approximate rise time of $8 \mathrm{nsec}$ and a maximum voltage magnitude of $25 \mathrm{kV}$. Predicted electrical design and performance of the line will be presented

\section{APPLICATION OF THE LINE}

The line will be used to provide transit time isolation of the reflections expected to be generated in the kicker of a multiple line-of-sight pulsed accelerator[1]. The kicker will operate with beam currents of 3 to $6 \mathrm{kA}$ and will launch beam-induced voltages up the transmissions lines connecting it to its pulsers. The most straightforward way to eliminate the effects of these reflections is to transit time isolate the kicker from the pulser. Thus the 10 $\mu \mathrm{sec}$ line will actually allow a $20 \mu \mathrm{sec}$ operational interval.

\section{WHY USE SUPERCONDUCTORS?}

A $10 \mu \mathrm{s}$ line, assuming waves traveling at the speed of light, corresponds to a line $3 \mathrm{~km}$ long. Needless to say, the magnitude of the problem requires a very low-loss line.

\subsection{Attenuation}

To demonstrate, let's look at the attenuation of a transmission line. The general expression for attenuation is

$$
\alpha=\frac{1}{2} \frac{R_{s}}{Z_{0}} \sqrt{\frac{\omega}{2}}
$$

where Rs is the skin-effect impedance dependent on the geometry and resistivity of the structure[2].

For a coaxial line the characteristic impedance is

$$
Z_{0}=\frac{\eta}{2 \pi} \ln (b / a)
$$

where $\eta$ is the impedance of free space $(377 \Omega)$ and $a$ and $b$ are the inner and outer diameter of the coax respectively. $R_{S}$ is [2][3]

$$
R_{s}=\frac{\sqrt{j \omega} \mu}{2 \pi} \Gamma,\left(\frac{1}{a}+\frac{1}{b}\right)
$$

$\Gamma_{r}$ is associated with the skin depth and is

$$
\Gamma_{r}= \begin{cases}1 / \sqrt{j \omega \mu \sigma} & \text { normal conducting metal } \\ 1 / \sqrt{j \omega \mu \sigma+1 / \lambda^{2}} & \text { Type I superconductor }\end{cases}
$$

where $\lambda$ is the penetration depth of the superconductor.

Suppose the line was made of copper $\left(\sigma=5.92 \mathrm{e} 7(\Omega-\mathrm{m})^{-1}\right)$ and the inner and outer diameters were $2 \mathrm{~cm}$ and $3 \mathrm{~cm}$ respectively. With an $8 \mathrm{nsec}$. rise time, we used $125 \mathrm{MHz}$ to be the highest critical frequency, which is about 3 times that of the roll-off frequency. For $125 \mathrm{MHz}, \alpha=7.658 \mathrm{e}-4 / \mathrm{m}$. For a $3 \mathrm{~km}$ line, this corresponds to a power attenuation of $\left(1-e^{-20.3000 m}\right)=98.9 \%$. 
On the other hand, for the same set of dimensions, a niobium $\left(\lambda=39 \mathrm{~nm}^{\prime}, \sigma=8.03 \mathrm{e}(\Omega-\mathrm{m})^{-1}\right)$ line yields an $\alpha$ of approximately $5.1025 \mathrm{e}-6(1+j) / \mathrm{m}$. The imaginary term contributes to the inductance of the line. The power attenuation is now $3 \%$ at $125 \mathrm{MHz}$ which is an upper limit. This is the most compelling reason to use a superconducting line.

\section{DESIGN OF A SUPERCONDUCTING LINE}

It is clear that attenuation is not an issue with these transmission lines. As it turns out, dispersion becomes the dominant issue, especially at higher frequencies. In addition, a good design should accommodate manufacturing considerations. The size of the transmission line is fundamentally limited by the critical field of the superconducting material. Of the type I conductors, niobium $(\mathrm{Nb})$ is the most generous material since it has the highest critical $B$-field of $206 \mathrm{~T}^{\prime}$ at $T=0 \mathrm{~K}$.

Assuming the distribution for the critical field to be

$$
B_{c}(T)=B_{c}(0)\left[1-\left(T / T_{c}\right)^{2}\right] \text {, }
$$

the critical field at $4.2 \mathrm{~K}$ (the usual operating point of liquid helium) is . $1635 \mathrm{~T}$. The $B$-field of a structure can be written as $B=g /$ where $g$ is a geometry factor. The condition on $g$ is therefore $g \leq B_{c} / /$

\section{I Coaxial line}

For a concentric coax, $g=\mu / 2 \pi r$ where $r$ is either the inner or outer radius of the line. This yields the condition that $r>\mu l / 2 \pi B_{c}$. If one wants to operate at $20 \%$ of maximum capacity, then $a=2 \mu l / 2 \pi B_{c}$ where $a$ is the inner radius. The current on the line can be as high as $1 \mathrm{kA}$. This requires that $a=6.12 \mathrm{~mm}$.

Since the layer of niobium needs only to be much greater than the penetration depth $\lambda$, a thin coat of approximately .Sum will be deposited on a copper substrate as shown in Fig. 1. The area between the two conductors will be filled with a material that may or may not be dielectric. The inner conductor should be hollow to allow helium to flow through the tube.

Design 1: The outer conductor radius, for an impedance of $25 \Omega$, is $9.28 \mathrm{~mm}$. Design 2: If one puts in a dielectric material of $\varepsilon_{r}=10$, the outer radius goes up to $22.85 \mathrm{~mm}$. The advantage of the dielectric is that the length of the line is reduced by a factor of $1 / \sqrt{10}$ which yields a length of $948 \mathrm{~m}$.

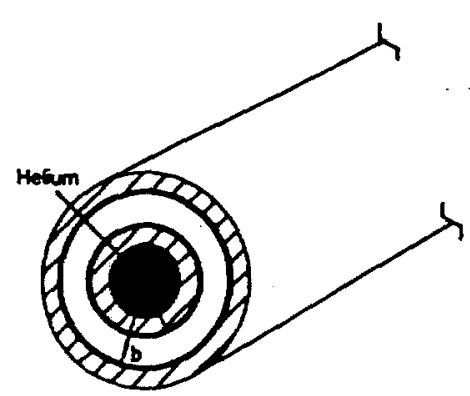

a)

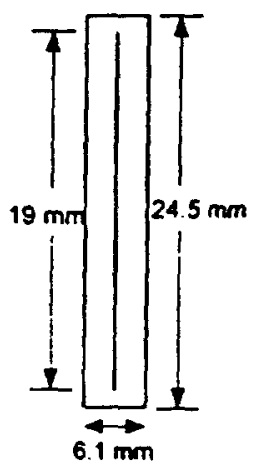

b)

Fig. I a) Coaxial line with $\mathrm{Nb}$ layers deposited on copper tubes. b) Dimensions of stripline design.

\subsection{Stripline}

For manufacturing considerations, it may be easier to fabricate striplines instead of coaxial lines. The solution for the stripline problem, both open and closed, has been derived by Primozich[4]. As it tums out, a stripline with a dielectric filler is too dispersive (see section 5). Therefore, the design cannot have a dielectric material. Design 3 : The stripline dimensions are shown in the following.

\footnotetext{
I see [2] back cover
} 


\subsection{Sheath helix}

Sheath helix lines are like coaxial lines, only the inner conductor is wrapped at an angle. These are slow wave structures and ideal for a delay line. However, they are more dispersive than regular coax. Fig $\mathcal{X}^{2}$ shows two sets of two overlayed $10 \mathrm{kV}$ pulses. The solid line is the actual signal at the output of the line and the dotted is the output had there been no dispersion. These plots are derivations based on Lund[5] and are for uncurved lines only.

Design 4: Fig $\vec{b}(\mathrm{a})$ corresponds to a line with $\varepsilon_{r}=1, b / a=1.15, p($ pitch $) / a=2.1$, and $v / c=.336$. The line length is reduced to about $1 / 3$ of the original length. Part (b) corresponds to $\varepsilon_{r}=3, b / \sigma=1.4, p / \sigma=2.75$, and $v / c=.264$. The second line yields a slightly slower wave but at the cost of increased dispersion as evident by the wiggles at the rising part of the pulse.

The sheath helix line is presented here as a backup design in case the stripline is not easy to manufacture because of it's length or the dielectric used for the coaxial line proves to be lossy or difficult to work with.
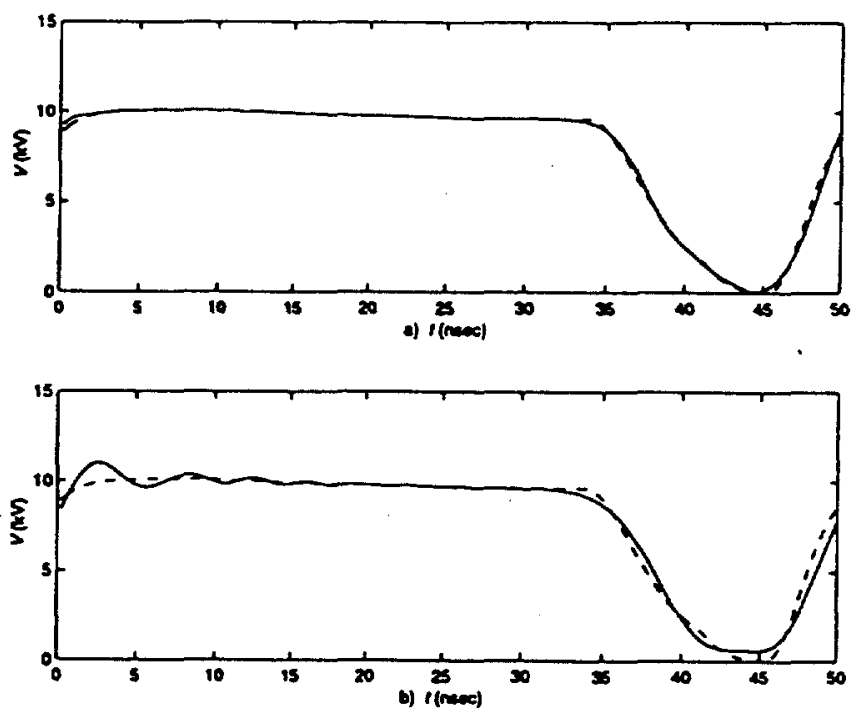

Fig. 2 a) - $10 \mathrm{kV}$ output pulse for sheath helix without dielectric, --- same pulse if line were non-dispersive b) With dielectric of 10 . Wiggles indicate dispersion.

\section{JOINT DESIGN}

Sections of the line need to be connected with superconducting joints of comparable geometry. An outer and inner sleeve, coated with $\mathrm{Nb}$, is clamped to the outer and inner conductors of the line. The design is shown in fig. 3. The wall thicknesses are uniformly $1 \mathrm{~mm}$.

Nb layer on Cu substrate

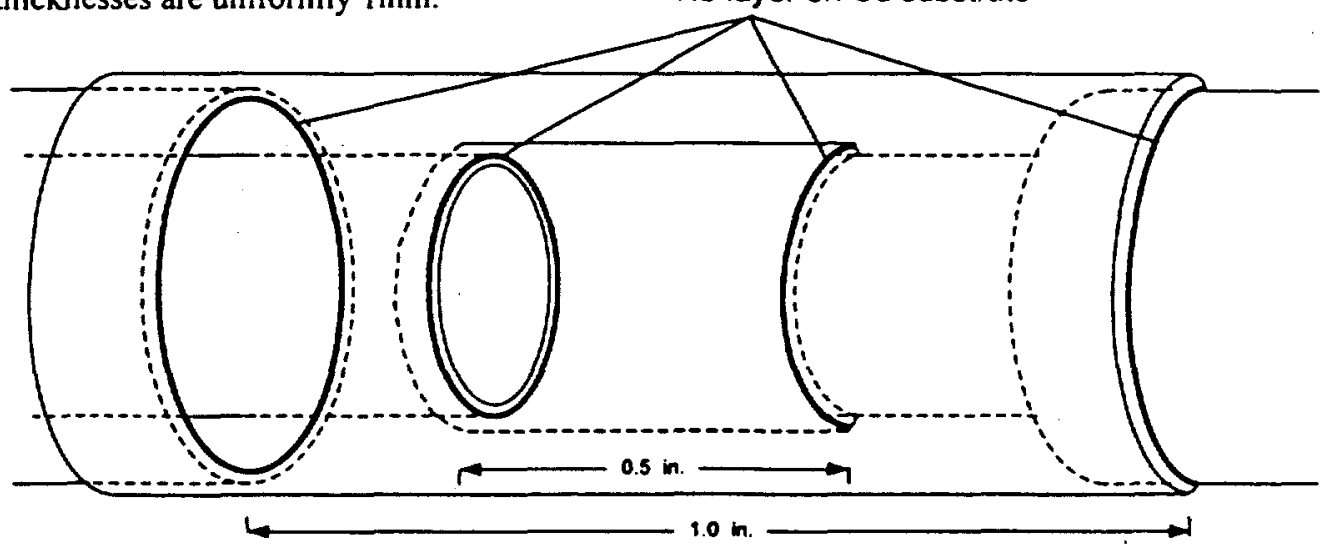

Fig. 3 Design shows ends of inner and outer conductors of coax sections connected with superconducting sleeves. 
The 3D finite element frequency domain solver MAXWELL EMINENCE ${ }^{2}$ was used to characterize the joint with $10 \mathrm{~cm}$ section coax (with no dielectric) connected on both ends. The impedance matrix from the numerical code was used to derive a voltage-current $(A B C D)$ matrix which can then be cascaded to model the entire line. In order to achieve an equivalent model for the line as shown in fig.4, the effective matrix is the product of the $A B C D$ matrices for both the joints and the sections of line. In other words,

$$
\left[\begin{array}{ll}
A & B \\
C & D
\end{array}\right]=\left[\begin{array}{ll}
A_{l} & B_{l} \\
C_{l} & D_{l}
\end{array}\right]\left[\begin{array}{ll}
A_{j} & B_{j} \\
C_{j} & D_{j}
\end{array}\right]\left[\begin{array}{ll}
A_{l} & B_{l} \\
C_{l} & D_{l}
\end{array}\right] \cdots\left[\begin{array}{cc}
A_{l} & B_{l} \\
C_{l} & D_{l}
\end{array}\right]
$$

where the subscript $l$ denotes line parameters, and subscript $j$ denotes joint parameters. $A=Z_{11} / Z_{12}, B=\left(Z_{1}\right)^{2}$. $\left.Z_{12}{ }^{2}\right) / Z_{12}, C=1 / Z_{12}, D=Z_{11} / Z_{12}$.

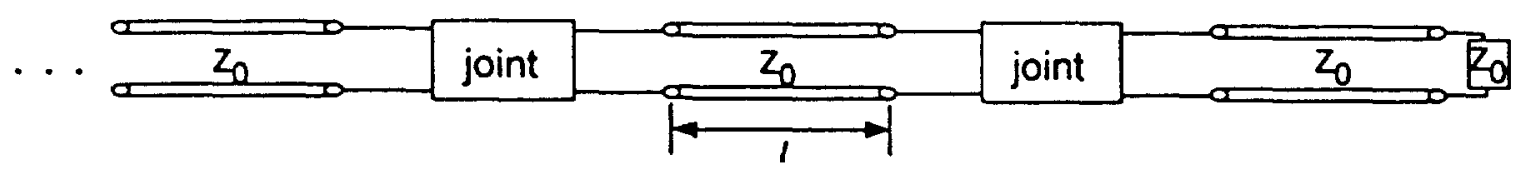

a)

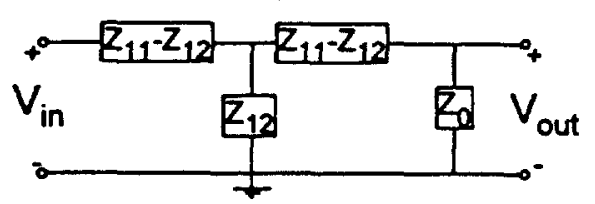

b)

Fig. 4 a) Block diagram of line and b) it's circuit model.

The impedance matrix of the transmission line portion is simply

$$
\begin{aligned}
& Z_{11}=-j Z_{0} \cot \gamma \\
& Z_{121}=Z_{11 /} / \cos \gamma
\end{aligned}
$$

The impedance matrix for the joint, as outputted from MAXWELL, leads naturally to equivalent circuit parameters. $Z_{12 j}$ is associated with a capacitance and $Z_{11 j^{-}} Z_{12 j}$ is associated with an inductance. At low frequencies, $Z_{11 j^{-}}$ $Z_{12 j}$ gives negative results which is unphysical. This is largely a numerical anomaly since it is the result of subtracting two large numbers of comparable values. The impedance values lie outside the error tolerance of the code. Therefore, only the linear part of the curve, between $100-250 \mathrm{MHz}$ is used to derive an effective inductance. The two curves yield $L_{j}=2.005 \mathrm{nH}$ and $C_{j}=6.104 \mathrm{pF}$. This yields a characteristic impedance $Z_{j}=V_{2} L_{j} / C_{j}=25.63 \Omega$, slightly higher than $Z_{O}$, the line impedance. The final circuit is shown in fig. $4 \mathrm{~b}$. The impedance parameters are extracted from the $A B C D$ matrix.

The output voltage pulses for a line with joints and one without joints are shown for a $3000 \mathrm{~m}$ line with $9.8 \mathrm{~m}$ sections and 300 joints. As one can see, the dispersion is barely perceptible. There is a slight shift in the output between the two cases due to the fact that the joints themselves introduce a small extraneous length. (Fig 5 )

\section{DISPERSION}

The dispersion property of conventional coaxial lines and striplines stems from a frequency dependent resistance (skin depth). The previous section has already shown that the effect from skin depth is negligible for a superconducting line. The dispersion on the superconducting transmission line, instead, is caused by wrapping the long line onto either a drum or into a pancake shape. The electromagnetic problem for a curved coax has been solved in [7].

\footnotetext{
2 crealed by ANSOFT
} 


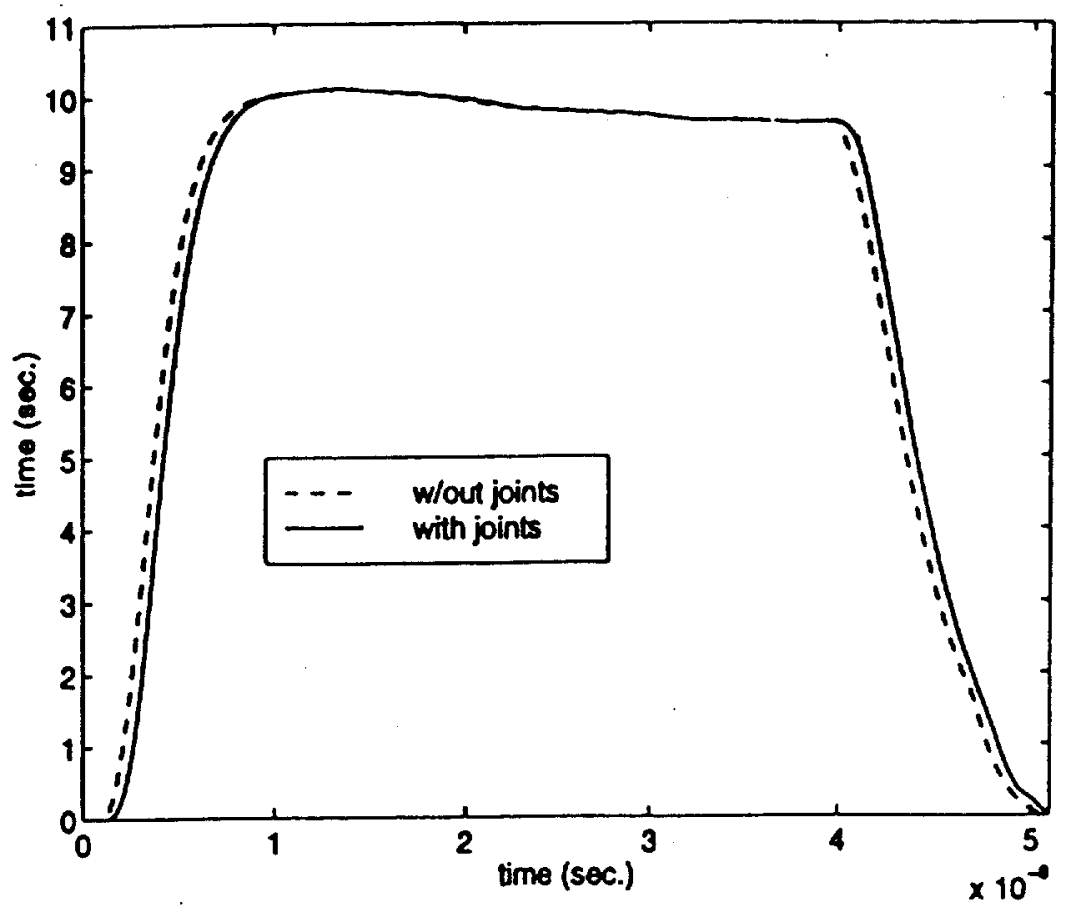

Fig. 5 Comparison of output between line with and without joints.
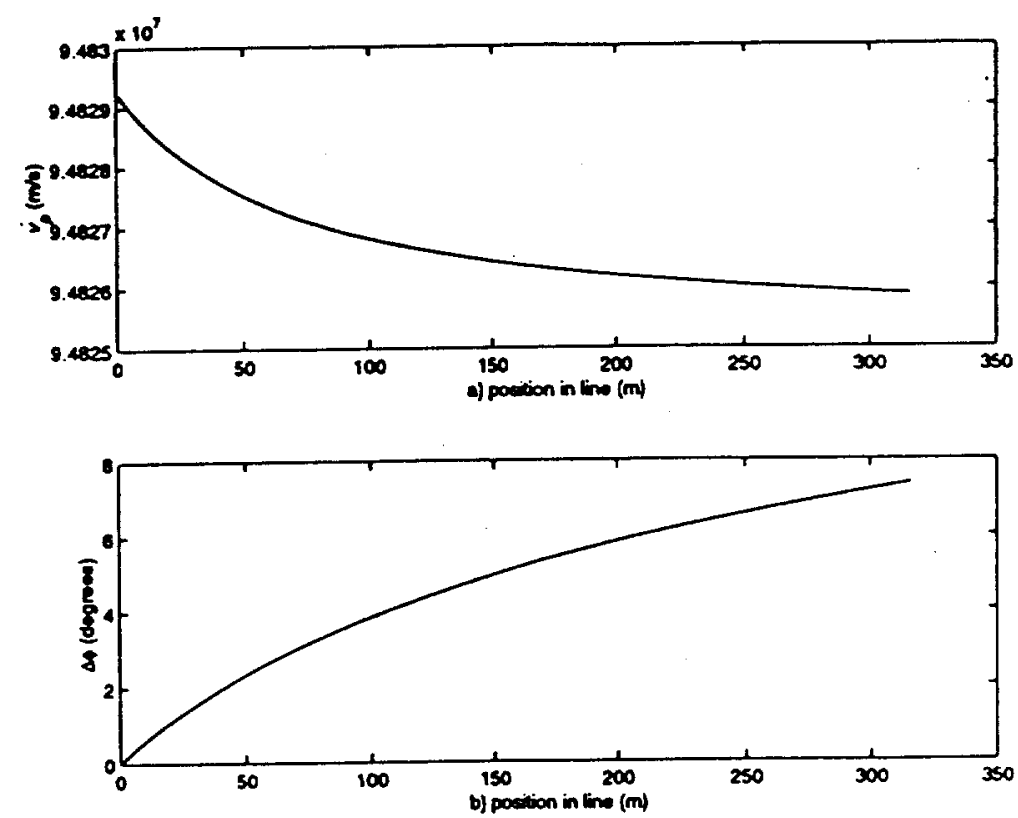

Fig. 6 a) Phase velocity vs. position in a pancake-wound coax line. b) It $s$ corresponding phase shift at $125 \mathrm{MHz}$. The propagation constant for a curved coax is as follows 


$$
\gamma^{2}=\gamma_{0}^{2}\left[1+\frac{A_{2}}{R^{2}}+O\left(\frac{1}{R^{4}}\right)\right]
$$

where $\gamma_{0}=\omega \sqrt{\mu \varepsilon}, A_{2}=A \omega^{2}+B$, and $R=R(s)$. The constants $A$ and $B$ can be found in [7]. For a pancake design, the outer cable radius is, as a function of $s$, the total length, and $R_{0}$, the inner radius,

$$
R(s)=\sqrt{R_{0}^{2}+\frac{2 b s}{\pi}}
$$

If one wants to find the total dispersion at the end of the line, the propagation constant would need to be integrated over the length of the line since it varies with $s$. In other words,

$$
e^{-j n} \rightarrow e^{-j j x e r}
$$

Therefore, the phase shift of the signal at the end of the line is

$$
\Delta \phi=-\frac{A_{2}(\varpi)}{2} \gamma_{0} \int_{0}^{s} \frac{d s^{\prime}}{R^{2}\left(s^{\prime}\right)}=-\gamma_{0} \frac{\pi A_{2}}{4 b} \ln \left(1+\frac{2 b s}{\pi R_{0}^{2}}\right)
$$

The phase velocity and $\Delta \phi$ for design 2 is plotted in Fig. 6. As one can see in Fig. 6 b, the phase distortion is less than $10^{\circ}$ at the end of the line for a frequency of $125 \mathrm{MHz}$. The striplines were treated as equivalent coax lines to estimate the dispersion due to bending.

The parameters for designs 1-3 were run through a kicker dynamic code to examine the acceptability of the output signal. All 3 designs produced clean output signals. There was a fifth design - a stripline with $\varepsilon_{r}=10-$ that yielded excessive dispersion.

\section{CONCLUSION}

Attenuation in long transmission lines made use of superconductors a necessity. The attenuation in the superconducting line is very small, with joints connecting sections of the line contributing to most of it. Dispersion, on the other hand, can arise simply from the geometry of the line. Curved lines and helix lines are subject to dispersion. Two coaxial lines, one stripline, and a sheath helix design were presented. The challenge of fabricating the line will determine which design is ultimately adopted.

\section{ACKNOWLEDGEMENTS}

The authors wish to acknowledge the technical support of Michael Krogh of AlliedSignals, Brian Poole, and Steve Sampayan. This work was performed under the auspices of the U.S. Department of Energy by the Lawrence Livermore National Laboratory under Contract No. W-7405-Eng-48.

\section{REFERENCES}

[1] 'Linear Induction Accelerator Approach for Advanced Radiography', G. Caporaso, to be published in the 1997 PAC proceedings.

[2] 'Transmission lines for digital and communication networks', by R.E. Matick, IEEE PRESS, 1995.

[3] 'Principles of Supercondutive Devices and Circuits,' by T. Van Duzer and C.W. Turner, published by Edward Amold, Ltd., 1981.

[4] F.G. Primozich, AIEE, May 1955.

(5) C.O. Lund, RCA Review, March 1950.

[6] 'Superconducting Magnets,' by M.N. Wilson, published by Oxford University Press, 1983.

[7] J.J. Krempasky, IEEE Transactions-MTT, v38, n6, June 1990. 


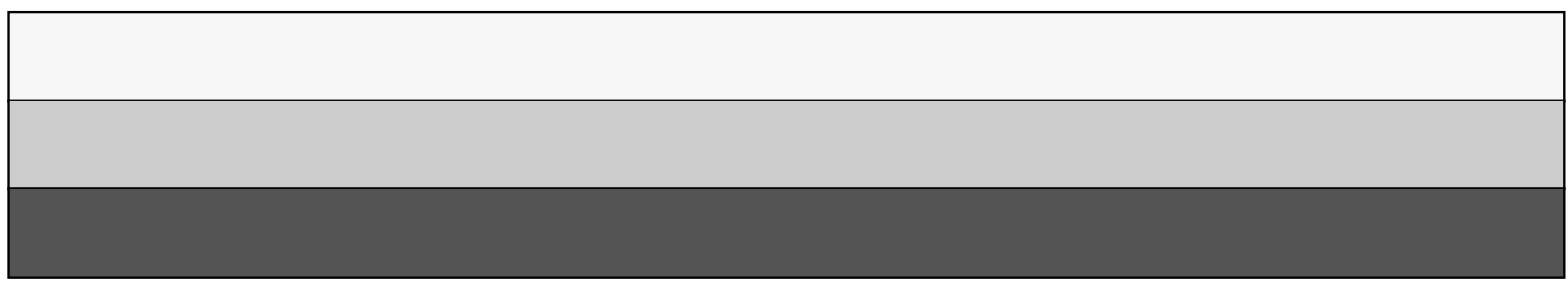

